\title{
BIBECHANA
}

ISSN 2091-0762 (Print), 2382-5340 (Online)

Journal homepage: http://nepjol.info/index.php/BIBECHANA

Publisher: Department of Physics, Mahendra Morang A.M. Campus, TU, Biratnagar, Nepal

\section{Modulation frequency and velocity variation of ions in a magnetized plasma sheath for different obliqueness of the field}

\author{
Bhesha Raj Adhikari ${ }^{1,2, *}$, Hari Prasad Lamichhane ${ }^{1}$, Raju Khanal ${ }^{1}$ \\ ${ }^{1}$ Central Department of Physics, Tribhuvan University, Kirtipur, Kathmandu, Nepal \\ ${ }^{2}$ Department of Physics, Bhaktapur Multiple Campus, Tribhuvan University, Bhaktapur, Nepal \\ * Email: b.r.adhikari@ hotmail.com
}

\section{Article Information:}

Received: May 29, 2020

Accepted: August 1, 2020

Keywords:

Bohm criterion

Kinetic theory

Sheath

Presheath

Modulation frequency

Damping constant

\begin{abstract}
The understanding of ion dynamics in magnetized plasma sheath is crucial for all applications of plasma. The velocity variation as well as modulation frequency of ions in a magnetized plasma sheath has been studied for different obliqueness of magnetic field. The governing Lorentz force equation has been solved numerically for the given boundary conditions as applicable in kinetic simulation of the sheath. For different obliqueness of the magnetic field, the average values, maximum amplitude, damping factor as well as frequency of oscillation are studied. The oscillating velocity components change at different rates depending on their orientation with respect to the field direction. Significant changes in the damping factor and modulation frequency has been observed for all components of velocity; however, the frequency of oscillation remains same. As the obliqueness increases, shoulder natures in the components of velocity are observed.
\end{abstract}

DOI: https://doi.org/10.3126/bibechana.v18i1.29171

This work is licensed under the Creative Commons CC BY-NC License. https://creativecommons.org/licenses/by-nc/4.0/

\section{Introduction}

The formation of non-neutral region in the vicinity of material wall is a ubiquitous feature of bounded plasma and it has wide range of growing applications in diverse fields (e.g. plasma confinement for fusion, sputtering, etching, surface treatment etc.) [1]. In plasma, the thermal velocity of electrons is higher as compared to that of the ions. Due to this, the wall is charged up negatively with respect to the core plasma. Therefore, negative potential attracts the ions and repels electrons forming a thin positive space charge region near to the wall. This positive space charge region, known as the 'sheath' separates the negatively charged material wall from the quasineutral 'presheath' plasma. The in-streaming ions have to satisfy the Bohm criterion to ensure the stability of the overall plasma $[2,3]$. The problem related to sheath is one of the oldest problems in plasma physics $[3,4]$ and once the plasma-wall interaction is well understood it will be possible to control heat loading, energy transfer and particle flow towards the wall and overall bulk plasma behavior [5 - 7]. 
The problem of sheath formation whenever it faces a material surface, its nature and evolution with time is significant for all plasma applications. This is a topic of interest and various works are reported recently as well; however, the study of modulation frequency as well as the time evolution of the ion velocity profile in a magnetized plasma sheath for varying obliqueness is still lacking [8-10]. This work focuses in the time variation of ion velocity, which shows oscillating nature, and its modulation frequency in a magnetized plasma sheath for different obliqueness of the field. The governing Lorentz force equation are formulated and solved numerically for the given boundary conditions as applicable in kinetic trajectory simulation (KTS) method of the sheath region $[8,11,12]$.

In typical boundary layer problems, the sheath region is of several electron-Debye lengths which is much smaller than the characteristic extension of the plasma. Such a sheath can only be formed, if the Bohm criterion [2, 5] is satisfied which demands that the ions enter the sheath region with a high velocity, which cannot be generated by thermal ion motion alone [5]. In the presence of a magnetic field, in kinetic form, the Bohm criterion reads

$\left\langle\frac{1}{v_{\|}^{2}}\right\rangle \leq \frac{1}{C_{s}^{2}}$

where,

$C_{s}=\sqrt{\frac{k\left(\gamma^{i} T_{P S}^{i}+\gamma^{e} T_{P S}^{e}\right)}{m^{i}}}$ is the ion-acoustic

velocity defined at the presheath side of the sheath edge, with $k$ is Boltzmann constant, $\gamma^{i}$ and $\gamma^{e}$ the ion and electron polytropic constants, respectively, $T_{p s}^{i}$ and $T_{p s}^{e}$ the ion and electron temperatures at the presheath side of the sheath edge, respectively and $m^{i}$ is the mass of ion species.

\section{Model and basic equations}

The $1 \mathrm{~d} 3 \mathrm{v}$ model of magnetized plasma sheath is shown schematically in Fig. 1. The region of interest is bounded by two parallel planes at $x=0$ and $x=L$, and the plasma consists of only electrons and singly charged ions. The plane at $x=L$ represents the "sheath entrance" that faces the plasma side and $x=0$ is the material wall, assumed to be non-emitting in the present case. The uniform magnetic field $\vec{B}$ acts on the $x y$-plane which makes an angle $\theta$ with the normal to the wall. In the presence of oblique magnetic field, the presheath consist of two distinct regions: collisional presheath and magnetic presheath [3, 13]. The general schematic diagram of magnetized plasma-wall transition region is shown in Fig. 2 [11].

As the sheath region is characterized by sharp gradients in a small scale of the order of electronDebye lengths, it is usually treated kinetically, where the particle distribution are governed by the Boltzmann equation [1]

$$
\frac{\partial f}{\partial t}+\vec{v} \cdot \nabla f+\frac{\vec{F}}{m} \cdot \nabla_{v} f=\left(\frac{\partial f}{\partial t}\right)_{c}
$$

where $\vec{F}$ is the macroscopic force acting on the particles, and $\left(\frac{\partial f}{\partial t}\right)_{c}$ is the time rate of change of distribution function $f(\vec{r}, \vec{v}, t)$ due to collisions. The symbol $\nabla$ represents the usual space-gradient operator whereas the symbol $\nabla_{v}$ represents the gradient in velocity space. For collisionless cases, suitable for sheath region as their dimension are much less than the mean free path, the equation (2) changes to so called Vlasov equation:

$$
\frac{\partial f}{\partial t}+\vec{v} \cdot \nabla f+\frac{q}{m}(\vec{E}+\vec{v} \times \vec{B}) \cdot \nabla_{v} f=0
$$

In the KTS method, the kinetic equations are solved along with other basic equations describing the plasma for given boundary and initial conditions. The distribution function at any point along the trajectory can be obtained if its value at one point (i.e., at the boundary) is known. Density of the species ' $s$ ' is then given by 


$$
n^{s}(\vec{x})=\int_{-\infty}^{+\infty} d^{3} v f^{s}(\vec{x}, \vec{v}),
$$

which then yields the space charge density as

$$
\rho(\vec{x})=\sum_{s} q^{s} n^{s}(\vec{x}) \text {. }
$$

Thus obtained space charge density is then used in the Poisson's equation to obtain the electrostatic potential $\phi(x)$

$$
\frac{d^{2} \phi(\vec{x})}{d x^{2}}=-\frac{\rho(\vec{x})}{\varepsilon_{0}},
$$

and the electric field is given by

$$
E(\vec{x})=-\frac{d \phi(\vec{x})}{d x}
$$

The kinetic equations (3)-(7) are solved along the collisionless trajectories for given boundary conditions. The ion velocity in the plasma sheath are computed using the Lorentz force equation

$m \frac{d \vec{v}}{d t}=q[\vec{E}+(\vec{v} \times \vec{B})]$

where $q$ is the charge of ion species whose mass is $m$.

The components of equation (8) are

$\frac{d v_{x}}{d t}=\frac{q E}{m}-\frac{q B v_{z} \sin \theta}{m}$

$\frac{d v_{y}}{d t}=\frac{q B v_{z} \cos \theta}{m}$

$\frac{d v_{z}}{d t}=\frac{q B v_{x} \sin \theta}{m}-\frac{q B v_{y} \cos \theta}{m}$

Since the magnetic field is dominating near the sheath entrance over the electric field, which is almost zero, the ions gyrate as they move slowly forward towards the wall. As the ions move towards the wall from the sheath entrance the electric field starts dominating, and hence the gyration decreases, and the equation of the damped harmonic oscillator can be is written as [14]

$$
v(t)=v_{m}+A e^{-k t} \sin (\omega t+\alpha)
$$

where $k, A, \omega$ and $\alpha$ are damping constant, amplitude, frequency of oscillation and phase angle, respectively. The damping constant of oscillating velocity components defined by equation (9) can be expressed as [14]

$k=\frac{\ln \left(\frac{v_{1}-v_{m}}{v_{2}-v_{m}}\right)}{t_{2}-t_{1}}$

where $v_{1}$ and $v_{2}$ are the resultant velocity at time $t_{1}$ and $t_{2}$, respectively and $v_{m}$ is the mean value of oscillating parts of velocity.

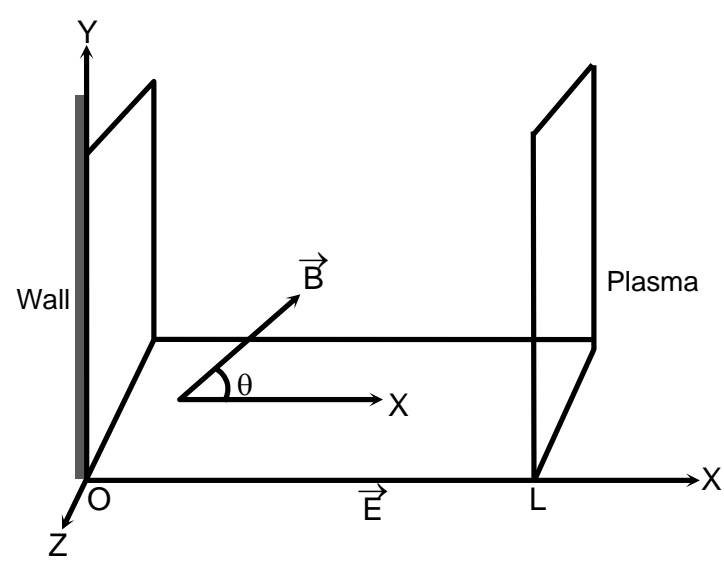

Fig. 1: Schematic geometry of the plasma-wall interaction model.

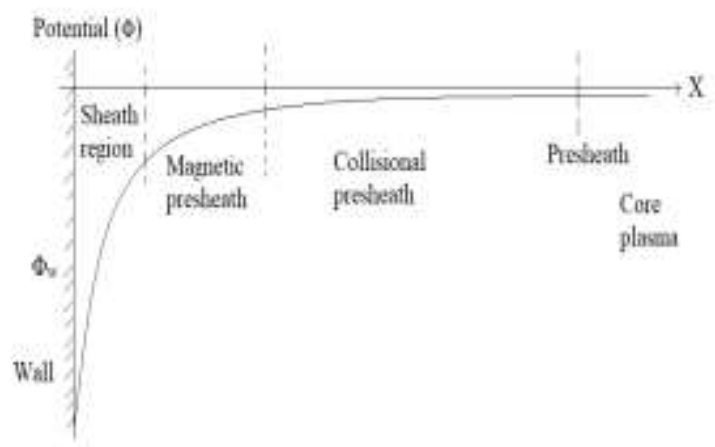

Fig. 2: Schematic diagram of magnetized plasmawall transition region.

\section{Results and Discussion}

In order to study the temporal variation of velocity, the equations (9)-(11) are solved by using RungeKutta method for the given boundary conditions. 
Furthermore, the amplitude of oscillation, damping factor, oscillation frequency and mean value are obtained by solving the equations (12) and (13) numerically. The temporal dependence of all three components of ion velocity have been calculated at constant magnetic field of $6 \mathrm{mT}$ and for three different obliqueness of the field $\left(30^{\circ}, 60^{\circ}\right.$ and $75^{\circ}$ ), shown in Figs. 3, 4, and 5, respectively. For the obliqueness of $30^{\circ}$, the $x, y$ and $z$-component of velocity varies about the mean value of $0.007 \mathrm{~m} / \mathrm{s}$, $6661 \mathrm{~m} / \mathrm{s}$ and $11600 \mathrm{~m} / \mathrm{s}$ respectively with same frequency of oscillation $100 \mathrm{~Hz}$ (Fig. 3). Also these components show damping nature of oscillation with damping factor $13.8 \mathrm{~s}^{-1}, 13.1 \mathrm{~s}^{-1}$ and $13.0 \mathrm{~s}^{-1}$, respectively. Likewise, these component of velocity varies with maximum amplitude of $9794 \mathrm{~m} / \mathrm{s}, 3138$ $\mathrm{m} / \mathrm{s}$ and $1550 \mathrm{~m} / \mathrm{s}$, respectively. It also shows that the modulation frequency of $x, y$ and $z$-component of velocity is same which is equal to $15.4 \mathrm{~Hz}$.

Fig. 4 shows that at obliqueness $60^{\circ}$, the $x, y$ and $z$ component of velocity varies about the average values of $-0.008 \mathrm{~m} / \mathrm{s}, 11570 \mathrm{~m} / \mathrm{s}$ and $6718 \mathrm{~m} / \mathrm{s}$, respectively with same frequency of oscillation, $100 \mathrm{~Hz}$. Also these components show damping nature of oscillation with damping factor of $15.2 \mathrm{~s}^{-}$ ${ }^{1}, 13.7 \mathrm{~s}^{-1}$ and $12.3 \mathrm{~s}^{-1}$ respectively. Likewise, these components of velocity vary with maximum amplitude of $9794 \mathrm{~m} / \mathrm{s}, 2650 \mathrm{~m} / \mathrm{s}$ and $4632 \mathrm{~m} / \mathrm{s}$, respectively. The modulation frequency of $x, y$ and $z$-component of velocity for this case is $16.2 \mathrm{~Hz}$, $15.8 \mathrm{~Hz}$ and $16.2 \mathrm{~Hz}$, respectively.

At obliqueness of $75^{\circ}$, the $x, y$ and $z$-component of velocity varies about the average values of 0.0058 $\mathrm{m} / \mathrm{s}, 11580 \mathrm{~m} / \mathrm{s}$ and $3137 \mathrm{~m} / \mathrm{s}$ respectively, with same frequency of oscillation $100 \mathrm{~Hz}$ as shown in Fig. 5. Also these components show damping nature of oscillation with the damping factor as $12.9 \mathrm{~s}^{-1}, 14.9 \mathrm{~s}^{-1}$, and $14.1 \mathrm{~s}^{-1}$, respectively. Likewise, these component of velocity varies with the maximum amplitude of $9794 \mathrm{~m} / \mathrm{s}, 1690 \mathrm{~m} / \mathrm{s}$ and $6657 \mathrm{~m} / \mathrm{s}$, respectively. The modulation frequency of $x, y$ and $z$-components of velocity are
15.8 Hz, $16.0 \mathrm{~Hz}$ and $15.4 \mathrm{~Hz}$, respectively. In this case, of obliqueness $75^{\circ}$, shoulder nature in the velocity components are observed around 0.05 second which was not the case when the angles were smaller.

The obtained results are summarized in Table 1, where the average value, maximum amplitude, damping factor, frequency of oscillation and modulation frequency of all three component of ion velocity at obliqueness of $30^{\circ}, 60^{\circ}$ and $75^{\circ}$. The $y$ component of mean value $(11580 \mathrm{~m} / \mathrm{s})$ is maximum at $75^{\circ}$ compared to other angles. The amplitude is maximum $(9793.99 \mathrm{~m} / \mathrm{s})$ of the $x$-component in two cases, when the angles are $30^{\circ}$ and $75^{\circ}$, whereas amplitude is minimum $(1550 \mathrm{~m} / \mathrm{s})$ for $z$-component at $30^{\circ}$. The damping factor of the $z$-component is minimum $\left(13.0 \mathrm{~s}^{-1}\right)$ when the angle is $30^{\circ}$, on the other hand it is maximum for $y$-component $\left(14.9 \mathrm{~s}^{-}\right.$ $\left.{ }^{1}\right)$ at $75^{\circ}$. Similarly, modulation frequency in the $x$ component and $z$-component is maximum (16.2 $\mathrm{Hz}$ ) at angle $60^{\circ}$, whereas frequency of oscillation remained same for all components of velocity for each cases considered.



Fig. 3: Temporal variation of velocity of ions for a magnetic field of $6 \mathrm{mT}$ at angle $30^{\circ}$. 


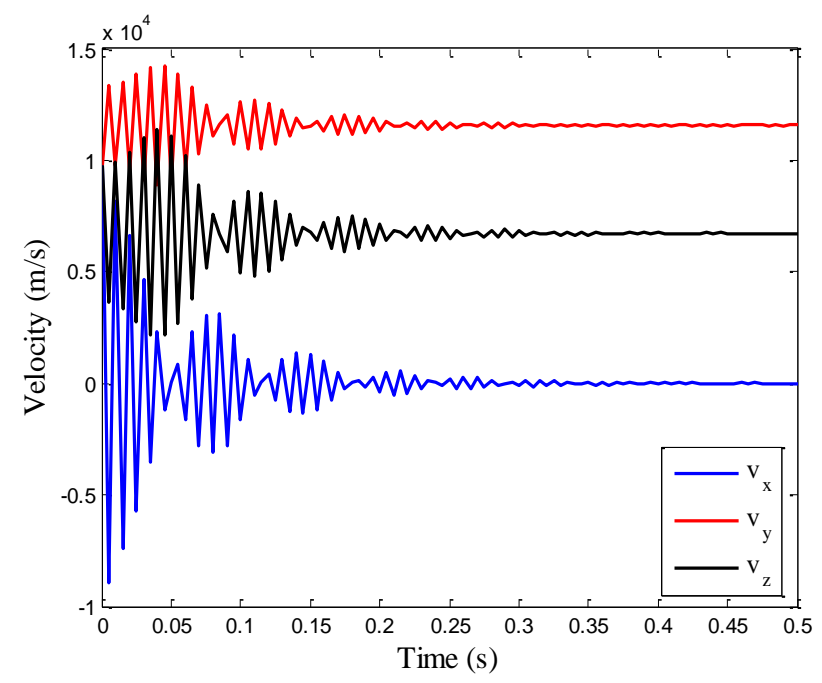

Fig. 4: Temporal variation of velocity of ions for a magnetic field of $6 \mathrm{mT}$ at angle $60^{\circ}$.

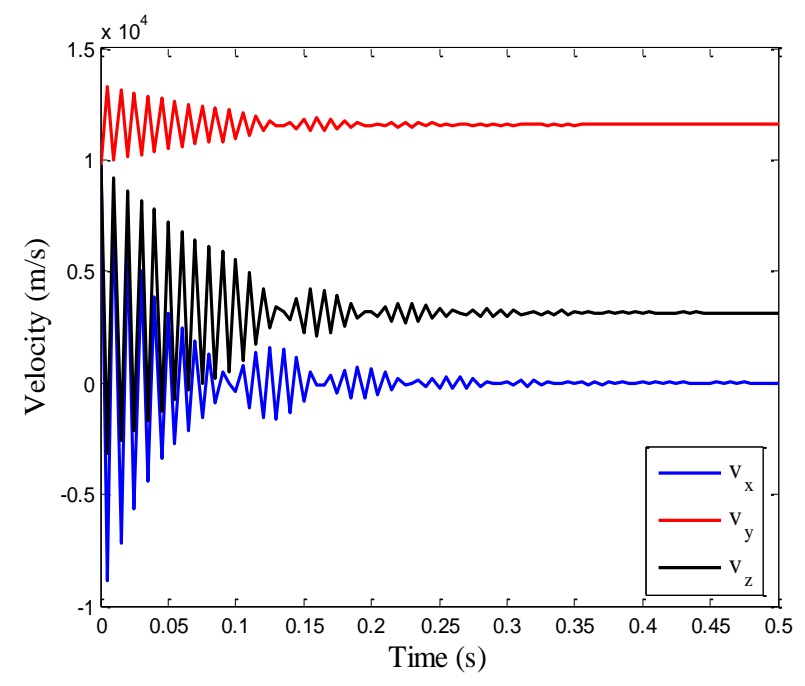

Fig. 5: Temporal variation of velocity of ions for a magnetic field of $6 \mathrm{mT}$ at angle $75^{\circ}$.

Table 1: The average value, maximum amplitude, damping factor, frequency of oscillation and modulation frequency of ion velocity

\begin{tabular}{|c|c|c|c|c|c|c|c|c|c|c|c|c|c|c|c|}
\hline \multirow[t]{2}{*}{$\theta$} & \multicolumn{3}{|c|}{$\begin{array}{c}\text { Average Value } \\
(\mathrm{m} / \mathrm{s})\end{array}$} & \multicolumn{3}{|c|}{\begin{tabular}{|c|} 
Maximum \\
Amplitude $(\mathrm{m} / \mathrm{s})$
\end{tabular}} & \multicolumn{3}{|c|}{$\begin{array}{l}\text { Damping } \\
\text { factor }\left(\mathrm{s}^{-1}\right)\end{array}$} & \multicolumn{3}{|c|}{\begin{tabular}{|c} 
Frequency of \\
oscillation \\
$(\mathrm{Hz})$
\end{tabular}} & \multicolumn{3}{|c|}{$\begin{array}{c}\text { Modulation } \\
\text { frequency }(\mathrm{Hz})\end{array}$} \\
\hline & $v_{x m}$ & & $\mathrm{~V}_{\mathrm{zm}}$ & $V_{x a}$ & $V_{\text {ya }}$ & $\mathrm{V}_{\mathrm{za}}$ & $V_{X}$ & $\mathrm{~V}_{\mathrm{y}}$ & $\mathrm{V}_{\mathrm{Z}}$ & $\mathrm{V}_{\mathrm{x}}$ & $\mathrm{V}_{\mathrm{y}}$ & $\mathrm{V}_{\mathrm{Z}}$ & $V_{X}$ & $\mathrm{~V}_{\mathrm{y}}$ & $\mathrm{V}_{\mathrm{Z}}$ \\
\hline $30^{\circ}$ & \begin{tabular}{|l|}
0.007 \\
\end{tabular} & 6661 & 11600 & 9793.99 & 3138 & 1550 & 13.8 & 13.1 & \begin{tabular}{|l|}
13.0 \\
\end{tabular} & 100 & 100 & 100 & 15.4 & 15.4 & 15.4 \\
\hline $60^{\circ}$ & -0.008 & 11570 & 6718 & 9794 & 2650 & 4632 & 15.2 & 13.7 & 13.3 & 100 & 100 & 100 & 16.2 & 15.8 & 16.2 \\
\hline $75^{\circ}$ & 0.0058 & 11580 & 3137 & 9793.99 & 1690 & 6657 & 12.9 & 14.9 & \begin{tabular}{|l|}
14.1 \\
\end{tabular} & 100 & 100 & 100 & 15.8 & 16 & 15.4 \\
\hline
\end{tabular}

\section{Conclusions}

Velocity variation as well as modulation frequency of ions in a magnetized plasma sheath has been studied for different obliqueness of magnetic field. It has been observed that the ion velocity at presheath-sheath boundary is affected by varying the obliqueness of the field. Various parameters like average ion velocity, amplitude, modulation frequency, and the damping factor can be controlled by varying the obliqueness. However, the frequency of oscillation is independent of obliqueness of magnetic field. The changes in these parameters results due to the change in contribution of the magnetic field with obliqueness. Present work is useful in understanding the time evolution of the particles velocities, and hence, exact particle behavior in magnetized plasma sheath region which is important in diverse plasma applications: material processing, surface treatment, plasma etching, confinement of plasma in fusion devices, and many more.

\section{Acknowledgement}

B. R. Adhikari would like to acknowledge the University Grants Commission, Nepal for the Ph. D. 
fellowship. The authors warmly thank Mr. Suresh Basnet for fruitful discussions.

\section{References}

[1] R. Chalise and R. Khanal, A kinetic trajectory simulation model for magnetized plasma sheath, Plasma Phys, Control. Fusion 54 (2012) 095006 (5pp).

https://doi.org/10.1088/0741-3335/54/9/095006

[2] D. Bohm, The characteristics of electrical discharges in magnetic fields, Edited by A. Guthry and P.K. Wakerling, MC-Graw Hill, 1949.

[3] R. Chodura, Plasma wall transition in an oblique magnetic field, Phys. Fluids 25 (1982) 1628-1633. https://doi.org/10.1063/1.863955

[4] I. Langmuir, Oscillations in ionized gases, Proc. National Academy of Sciences 14 (1928) 627637.

[5] K. U. Riemann, The Bohm criterion and sheath formation, J. Phys. D: Appl. Phys. 24 (1991) 493518. https://doi.org/10.1088/0022-3727/24/4/001

[6] K. U. Riemann, Theory of the plasma-sheath transition, J. Tech. Phys. 41 (2000) 89-121.

[7] M. M. Hatami, A. R. Niknam, B. Shokri and H. Ghomi, Magnetized plasma sheath with two species of positive ions, Phys. Plasmas 15 (2008) 053058 (5pp).

https://doi.org/10.1063/1.2918319
[8] B. R. Adhikari, S. Basnet, H. P. Lamichhane and R. Khanal, Presheath-sheath coupling for kinetic trajectory simulation of a magnetized plasma sheath, AIP Advances 9 (2019) 055123 (10pp). https://doi.org/10.1063/1.5088107

[9] R. Moulick, S. Adhikari and K. S. Goswami, Sheath formation in collisional, low pressure, and magnetized plasma, Phys. Plasmas 26 (2019) 043512 (10pp). https://doi.org/10.1063/1.5090537

[10] S. Basnet, A. Sarma and R. Khanal, Effect of presheath electron temperature on magnetized plasma-wall transition and wall sputtering by plasma having two species of positive ions, Physica Scripta 95(2020) $065601 \quad$ (11pp). https://doi.org/10.1088/1402-4896/ab7b89

[11] R. Khanal, A Kinetic trajectory simulation model for bounded plasma, Ph. D. Thesis. Innsbruck University, Austria (2003).

[12] R. Chalise and R. Khanal, Self consistent one dimension in space and three dimension in velocity kinetic trajectory simulation model of magnetized plasma wall transition, Phys. Plasmas 22 (2015) 113505 (5pp). https://doi.org/10.1063/1.4934601

[13] P. C. Stangeby, The plasma boundary of magnetic fusion devices, IOP Publishing Ltd ( 2000).

[14] G. F. Simmons, Differential equations with applications and historical notes, Tata McGrawHill, New Delhi (1991). 